\title{
Efficacy of Exercise Interventions in Patients with Acute Leukemia: A Meta-Analysis
}

\author{
Yuan Zhou ${ }^{1}$, Jinjie Zhu ${ }^{2}$, Zejuan $\mathrm{Gu}^{3 *}$, Xiangguang Yin ${ }^{4}$ \\ 1 The First Hospital Affiliated with Nanjing Medical University, Nanjing, Jiangsu, PR China, 2 College of \\ Aerospace Engineering, Nanjing University of Aeronautics and Astronautics, Nanjing, Jiangsu, PR China, \\ 3 Department of Nursing, The First Hospital Affiliated with Nanjing Medical University, Nanjing, Jiangsu, PR \\ China, 4 College of Nursing, Nanjing Medical University, Nanjing, Jiangsu, PR China \\ * jassicagu@163.com
}

\section{Abstract}

\section{Background}

Decreased physical performance and impaired physiological and psychological fitness have been reported in patients with acute leukemia (AL). We performed a meta-analysis to assess the efficacy of exercise in patients with AL.

\section{Methods}

In this meta-analysis, the electronic databases MEDLINE, Embase, Cochrane, Web of Science, SPORTDiscus, CINAHL and PEDro were searched through November 2015. Three authors participated in the study selection, data extraction and quality assessment. The instrument used for quality assessment was derived from the Cochrane Handbook for Systematic Reviews of Interventions. Analyses were performed according to the recommendations of The Cochrane Collaboration using Review Manager 5.3.

Editor: Alejandro Lucia, Universidad Europea de Madrid, SPAIN

Received: February 27, 2016

Accepted: July 9, 2016

Published: July 27, 2016

Copyright: @ 2016 Zhou et al. This is an open access article distributed under the terms of the Creative Commons Attribution License, which permits unrestricted use, distribution, and reproduction in any medium, provided the original author and source are credited.

Data Availability Statement: Data are from the literatures included in the paper.

Funding: This work was supported by Jiangsu Province, "333 Project" Scientific Research Project [2014] No. 28, http://www.jsrcgz.gov.cn/333gc/ gcindex.aspx.

Competing Interests: The authors have declared that no competing interests exist.

\section{Results}

Nine trials (8 randomized controlled trials and 1 quasi-experimental design trial) with $314 \mathrm{AL}$ participants were included in this meta-analysis. The pooled standardized mean differences between the exercise and control groups were 0.45 (95\% confidence interval $(\mathrm{Cl}): 0.09$ to $0.80, \mathrm{P}$ value $=0.01, \mathrm{P}$ for heterogeneity $=0.23, \mathrm{I}^{2}=28 \%$ ) for cardiorespiratory fitness and $0.67\left(95 \% \mathrm{Cl}: 0.28\right.$ to $1.06, \mathrm{P}$ value $=0.0007, \mathrm{P}$ for heterogeneity $\left.=0.14, \mathrm{I}^{2}=43 \%\right)$ for muscle strength. Based on the data for fatigue, anxiety, and depression, there were no significant differences in these parameters between the exercise and control groups.

\section{Conclusions}

Exercise has beneficial effects on cardiorespiratory fitness, muscle strength and functional mobility; however, no significant improvements in fatigue, anxiety, depression or quality of life were observed. Further large-scale randomized trials are needed to assess the safety, feasibility and efficacy of exercise programs for AL patients. 


\section{Introduction}

Cancer patients gradually exercise less as the disease progresses and typically get less than the recommended level of exercise, as stated in previously published studies [1,2]. This decline is caused by various factors, including cardiopulmonary, gastrointestinal and neurological toxicities, anemia, thrombocytopenia and cachexia [3]. Moreover, fatigue, anxiety, depression, fear and the risk of infection, along with the oncologist's recommendation of prolonged rest, may further limit a patient's exercise options [4]. A low level of exercise has been extensively reported to have negative impacts on cardiopulmonary and musculoskeletal function $[5,6]$, cognitive abilities [7], social function, and psychological well-being [8]. Therefore, exercise has been implemented in breast cancer patients to improve physical function and activities of daily living since the 1980s [9]. Most subsequent studies have intensively investigated exercise in relation to colorectal and prostate cancers, in addition to other types of solid tumors [10-12]. As an ancillary treatment to oncotherapy, exercise may improve quality of life (QOL), physical fitness, mood and many other factors in patients with solid tumors [13]. For hematologic cancers, some evidence has suggested that exercise may improve oxygenation and physical wellbeing, in addition to increasing muscle strength and cardiorespiratory fitness [4,14-16]. Therefore, exercise has gradually become integrated into cancer treatment programs because of its positive impacts.

Acute leukemia (AL) is a malignant hyperplastic disease of the hematopoietic system that is associated with high morbidity and that typically develops rapidly. Cancer statistics from 2016 [17] showed that among the 26,540 estimated new cases of AL in the United States, 11,860 deaths occurred. AL patients may feel quite fatigued, bruise easily and develop frequent infections, which are the main factors resulting in low levels of exercise among this population. As stated previously, a low level of exercise negatively affects physical and psychological function. Therefore, the efficacy of exercise as a component in the treatment of AL patients must be assessed.

Through a literature search, we identified only one systematic review (SR) on the effects of exercise in leukemia patients [16]. It is a descriptive review that lacks aggregated data and includes both acute and chronic leukemia subjects. Notably, the pathogeneses, clinical manifestations, treatments and other aspects differ between these two types of leukemia, and these differences were not considered in that review. Moreover, differences in survival rates and therapies between adults and children were not taken into consideration [18]. With the considerable growth in this research area, exercise studies of AL patients [19-21] that are not included in existing reviews have recently been published. Here, we performed meta-analysis to critically assess the effects of exercise on cardiorespiratory fitness, muscle strength, fatigue, anxiety, and depression in AL patients. In addition, subgroup analyses were performed to evaluate the effects of exercise in adult/pediatric patients and in those receiving induction/post-remission therapy.

\section{Materials and Methods}

\section{Inclusion criteria and search strategy}

The following inclusion criteria were applied: (1) population: human participants with a diagnosis of AL (either acute myelocytic leukemia (AML) or acute lymphoblastic leukemia (ALL)) undergoing induction therapy or post-remission therapy; (2) intervention: an exercise component, regardless of the type of exercise; (3) comparison intervention: standard care with no exercise intervention or instruction; (4) outcome measures: cardiorespiratory fitness, muscle strength, fatigue, anxiety, depression, QOL, hemoglobin level, body mass index (BMI) and 
functional mobility; and (5) study design: a randomized controlled trial (RCT) or a quasiexperimental design trial. To maximize the number of eligible studies for review, no restrictions were placed on sample size or age. Subgroup analyses were conducted based on adults/children and induction/post-remission therapy.

In this meta-analysis, seven databases, including MEDLINE, Embase, Cochrane, Web of Science, SPORTDiscus, CINAHL and PEDro, were searched through November 2015. This computerized search was performed using the following terms: (leukem* OR leukaem* ${ }^{*}$ AND (exercise OR exercise movement techniques OR training program* OR physiotherapy OR physical therap* modality OR physical endurance OR physical exertion OR physical fitness OR physical education and training OR physical activity $O R$ walking OR exercise therapy OR rowing* OR ramble* $^{*}$ OR rambling* OR danc* OR mountaineer* OR cycling* OR bicycling* OR swimming* OR jogging* OR ambulation* OR running* OR athletic ${ }^{*}$ OR sport ${ }^{*}$ OR stretching* OR yoga* OR tai chi OR tai ji OR ji quan tai OR train* OR pilates* OR calisthenic* OR gymnastic*) AND (trial OR randomized OR clinical trials OR placebo OR randomized OR controlled clinical trial OR randomized controlled trial). The search strategy combined text words with medical subject heading terms and was limited to human subjects. No language restriction was applied.

\section{Study selection and data extraction}

Two authors (Zhou and Zhu) independently screened the retrieved results that met the inclusion criteria by reading the abstracts and titles. If any question arose as to whether a study should be included, the full text was searched. In cases of disagreement, a third author (GU) joined the discussion to reach a consensus.

For each study, the following data were extracted by the reviewer (Zhou): the study details (first author's last name, year of publication, and type of study); participant characteristics (diagnosis, department, timing, sample size, age, gender, and location); intervention groups (frequency, intensity, time, type, and duration); comparison groups; outcome measured; follow-up times; and funding. All extracted data were verified by a second reviewer ( $\mathrm{Zhu})$. The extracted data was imported into a Microsoft Excel file, and the data were again verified by a third investigator $(\mathrm{Gu})$. Any discrepancies were resolved by discussion until a consensus was reached.

\section{Quality assessment}

The methodological quality and risk of bias of each trial were evaluated according to the Cochrane Handbook for Systematic Reviews of Interventions [22]. The following criteria were included: random sequence generation (selection bias); allocation concealment (selection bias); blinding of participants and personnel (performance bias); blinding of the outcome assessor (detection bias); incomplete outcome data (attrition bias); selective reporting (reporting bias); and other biases. The quality assessments were independently conducted (Zhou and Zhu), and any disagreements were resolved by discussion.

\section{Data analysis}

The data from the included studies were sufficient for conducting meta-analysis. Analyses were performed according to the recommendations of the Cochrane Collaboration, and Cochrane Statistical Package Review Manager 5.3 was used for analysis. For the continuous outcomes, we calculated the mean difference (MD) or standardized mean difference (SMD) with the $95 \%$ confidence interval (CI) for each study. Study heterogeneity was assessed using the $\mathrm{Chi}^{2}$ test at a significance level of $\mathrm{P}<0.1$, in addition to the $\mathrm{I}^{2}$ statistic $\left(\mathrm{I}^{2}>50 \%\right.$ indicated substantial heterogeneity) [22]. 


\section{Results}

\section{Search results}

A total of 963 articles were identified in the database searches; no additional records were identified through other sources. After deleting any duplicates, 788 articles remained. At the initial screening stage, 751 articles were excluded based on their titles and abstracts because they did not meet the predefined inclusion criteria. The full texts of the 37 remaining articles were read. Of them, 28 articles were excluded for various reasons. At the end of the screening procedure, 9 studies remained [19-21,23-28]. The screening details are presented in a PRISMA flow diagram (Fig 1).

\section{Study characteristics and risk of bias}

Nine trials were included in this meta-analysis [19-21,23-28]. Eight were RCTs, and 1 was a quasi-experimental design trial [28]. From these 9 studies, 314 participants were included in analysis ( 3 patients dropped out, 171 were males, 138 were females, and 2 were of unknown gender; 143 had AML, and 171 had ALL). The features of the included trials are summarized in Table 1. For a summary of each item associated with risk of bias, see Figs 2 and 3.

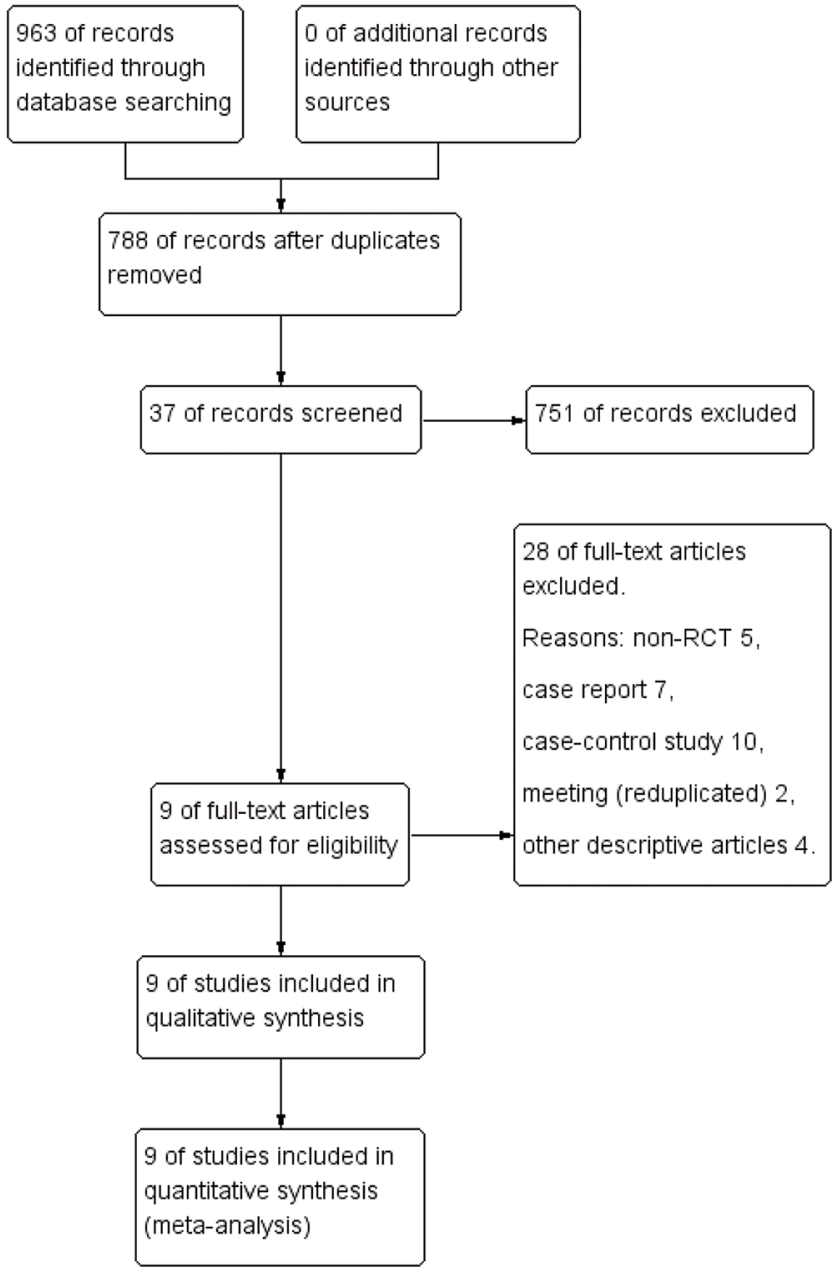

Fig 1. Flow diagram of the study selection process.

doi:10.1371/journal.pone.0159966.g001 


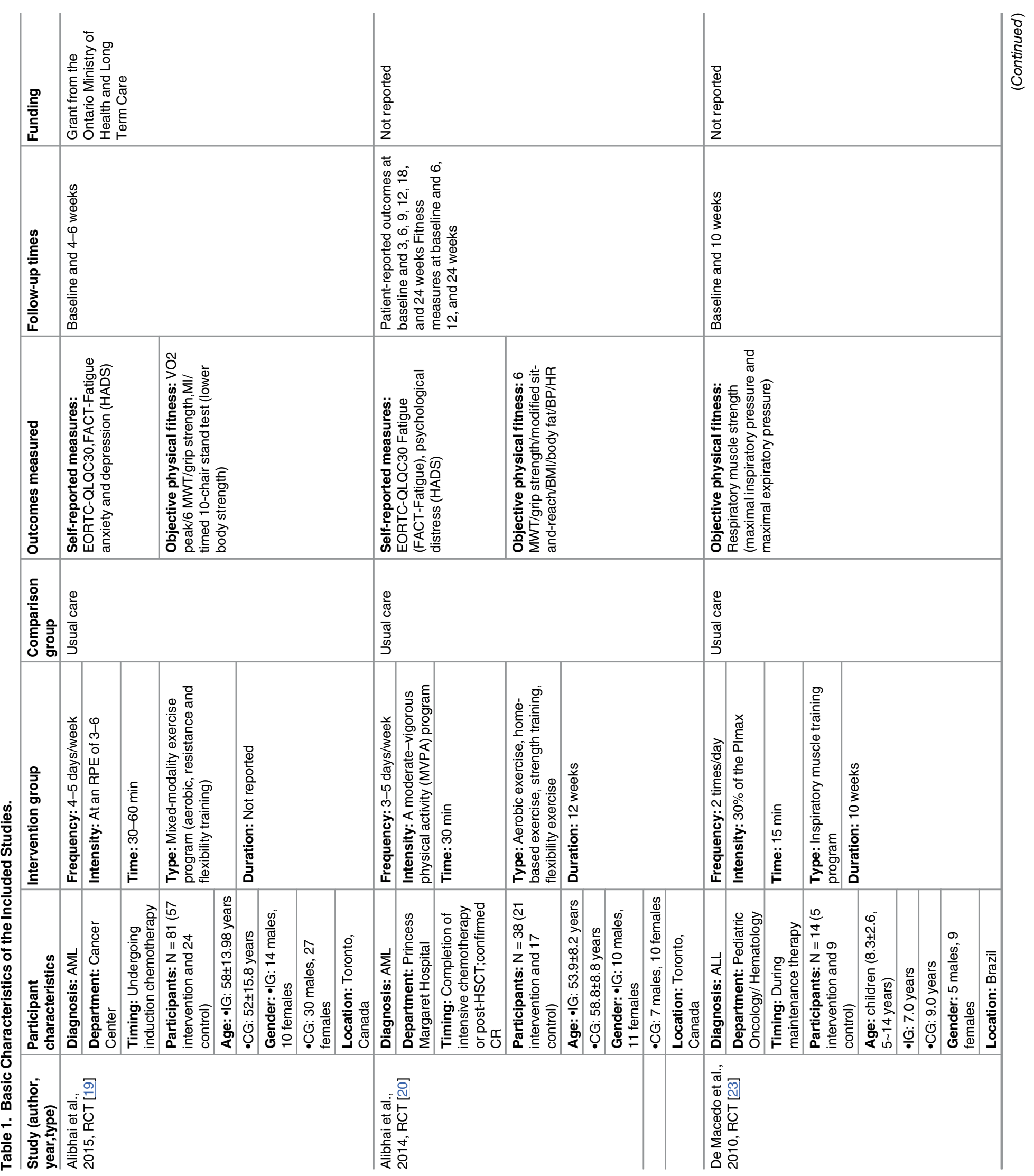




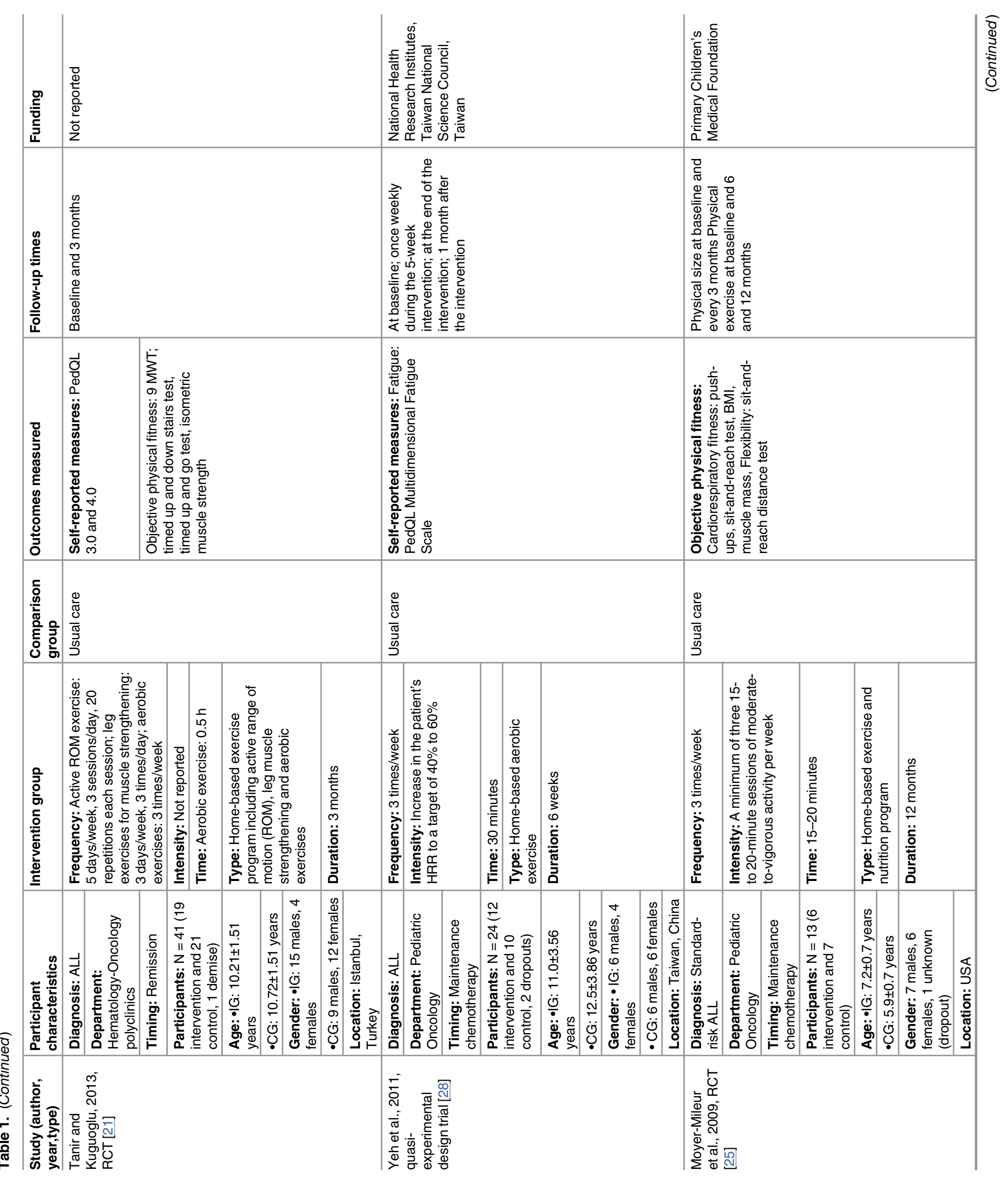




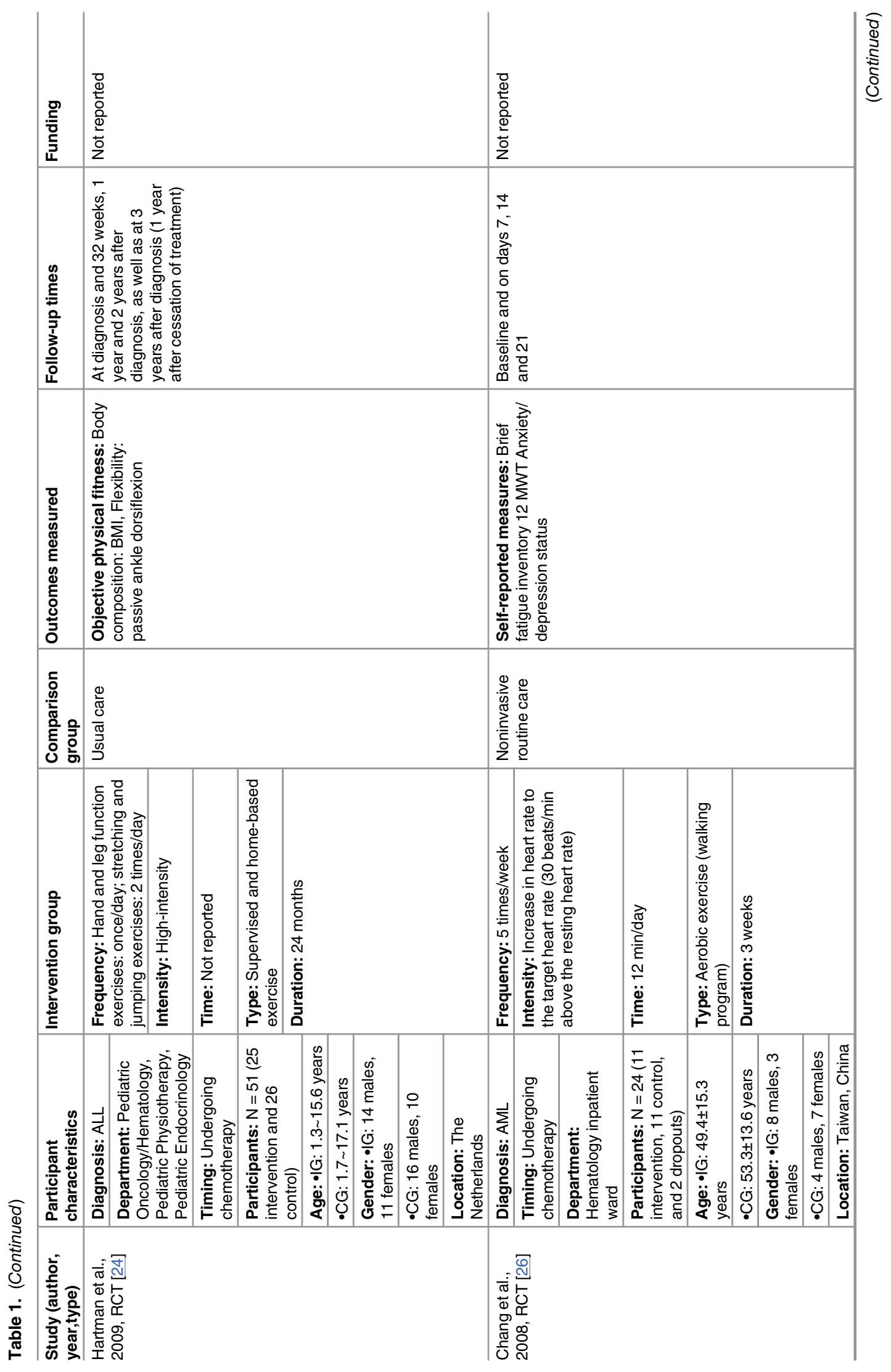




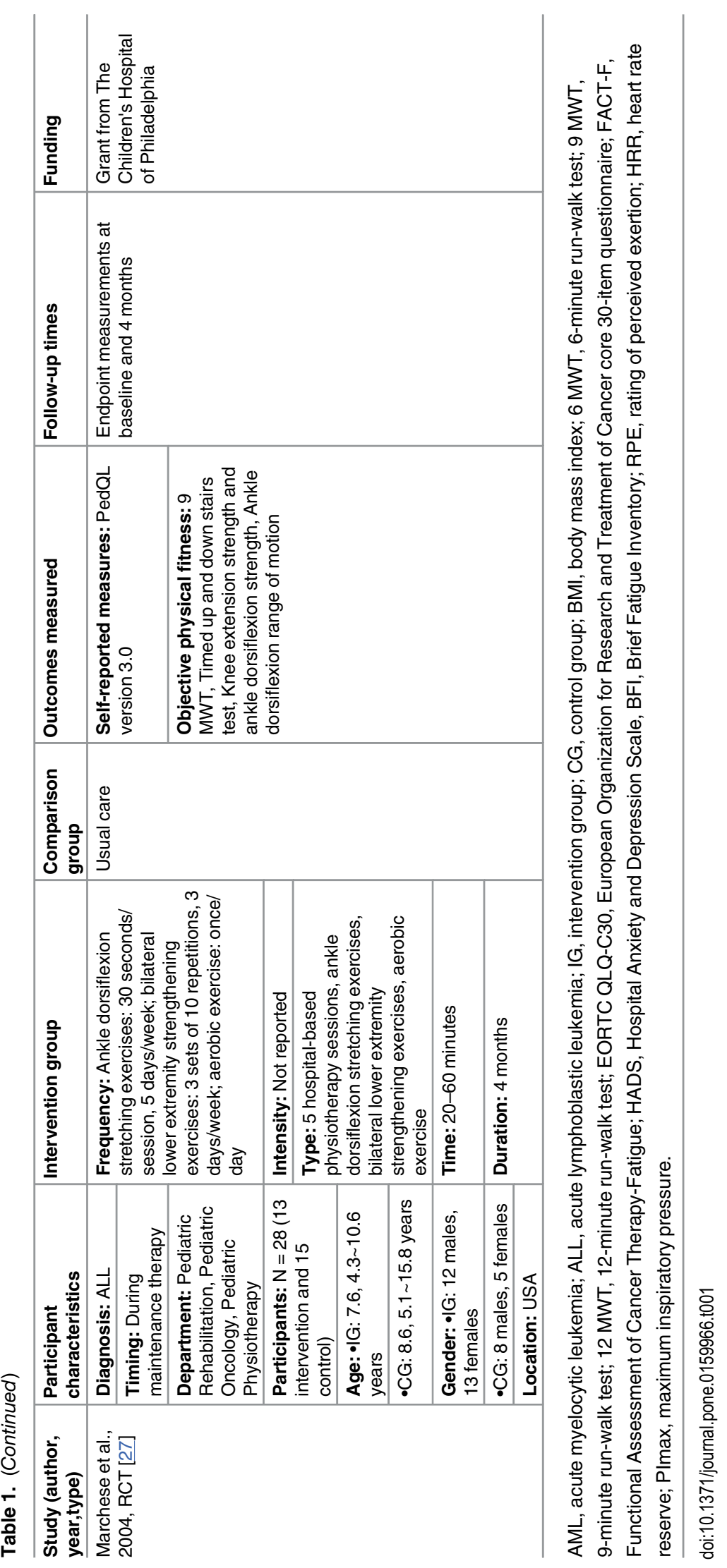




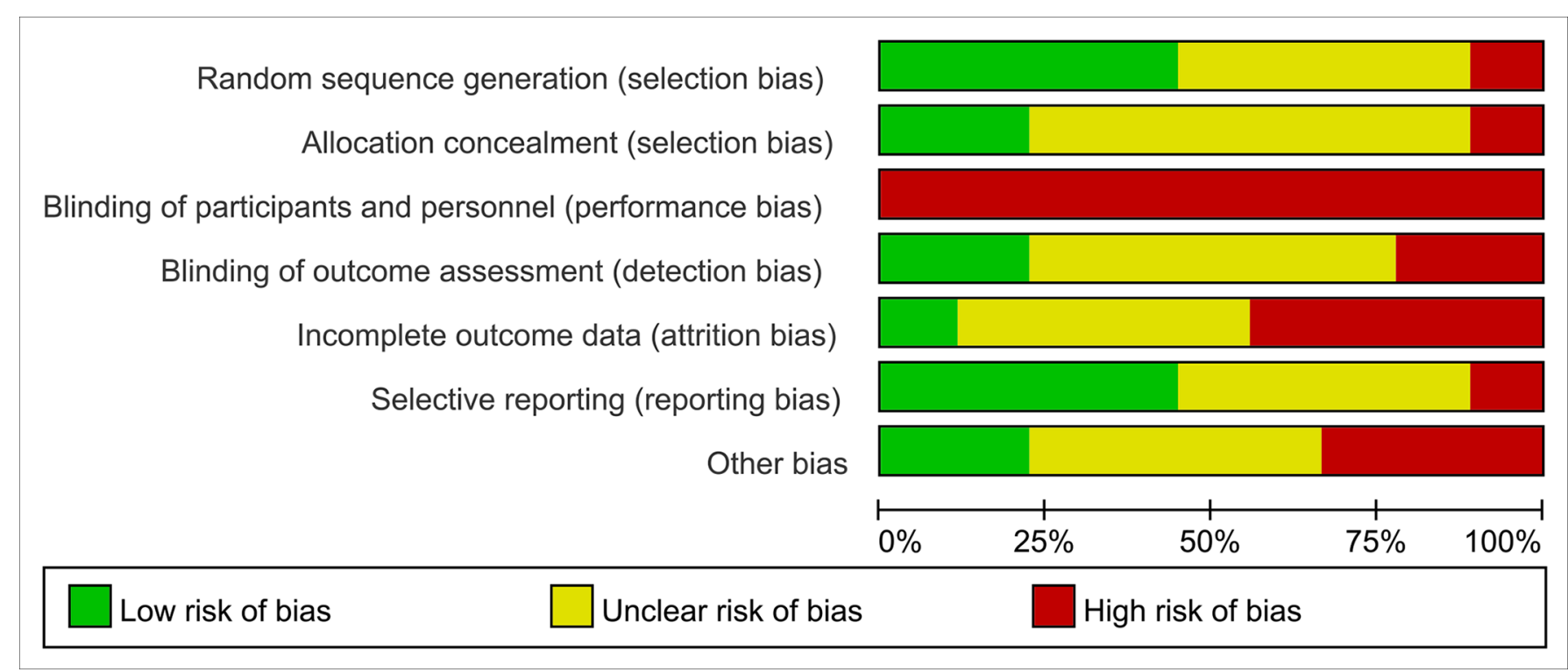

Fig 2. Risk of bias graph.

doi:10.1371/journal.pone.0159966.g002

\section{Meta-analysis of outcome measures}

Cardiorespiratory fitness. In five included trials $(n=196)$, cardiorespiratory fitness was assessed via run-walk tests, with varying durations between the trials [19-21,26,27]. Subgroup analyses were conducted on the 6-min, 9-min and 12-min run-walk test subgroups. The cardiorespiratory fitness of the 6-and 9-min subgroups did not significantly differ between the exercise and control interventions; however, the 12-min subgroup showed a significant improvement in cardiorespiratory fitness for the exercise intervention compared to the control intervention. The aggregated results of the five studies revealed a significant difference in cardiovascular fitness between the interventions (SMD $=0.45,95 \%$ CI: 0.09 to $0.80, \mathrm{P}$ value $=0.01$, $\mathrm{P}$ for heterogeneity $=0.23, \mathrm{I}^{2}=28 \%$ ). The details are presented in Fig 4.

Muscle strength. Four studies $(n=160)$ measured muscle strength in AL patients [1921,23]. Muscle strength was assessed via different measurements for different muscle groups. Alibhai et al. [19,20] measured grip strength using a Jamar dynamometer and lower body strength via the timed 10-chair stand test. Tanir and Kuguoglu [21] measured isometric muscle strength using a dynamometer. De Macedo et al. [23] measured respiratory muscle strength based on maximum inspiratory and expiratory pressure. The aggregated results of these studies showed a significant difference in muscle strength between the intervention and control groups, with an SMD of 0.67 (95\% CI: 0.28 to 1.06, $\mathrm{P}$ value $=0.0007$, $\mathrm{P}$ for heterogeneity $=0.14$, $\mathrm{I}^{2}=43 \%$ ) (Fig 5).

Fatigue, anxiety and depression. Four studies $(\mathrm{n}=160)[19,20,26,28]$ provided data on fatigue measurements. Fatigue was assessed by Alibhai et al. [19,20] using the Functional Assessment of Cancer Therapy-Fatigue subscale (FACT-Fatigue), by Chang et al. [26] using the Brief Fatigue Inventory (BFI), and by Yeh et al. [28] using the PedQL Multidimensional Fatigue Scale. The aggregated results of these studies showed no significant difference in fatigue between the groups ( $\mathrm{SMD}=-0.18,95 \% \mathrm{CI}$ : -0.53 to 0.16 , $\mathrm{P}$ value $=0.28$, $\mathrm{P}$ for heterogeneity $\left.=0.38, I^{2}=2 \%\right)$. Anxiety and depression were assessed by Alibhai et al. $[19,20]$ using the Hospital Anxiety and Depression Scale and by Chang et al. [26] using the depression and anxiety subscales of the Profile of Mood States Short Form. The results revealed non-significant differences in anxiety and depression between the groups (anxiety: SMD $=-0.22,95 \% \mathrm{CI}$ - -0.82 to 


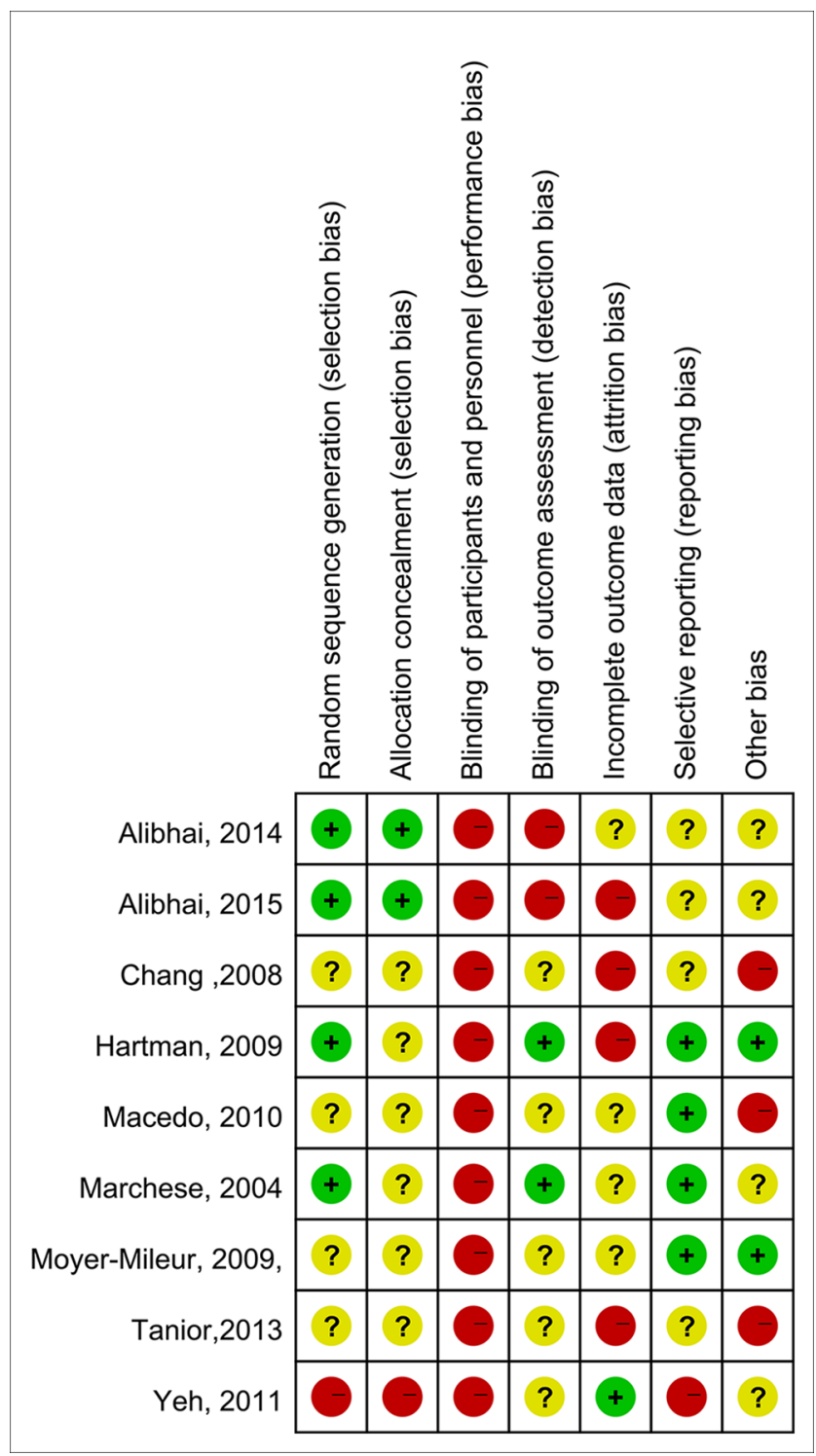

Fig 3. Risk of bias summary.

doi:10.1371/journal.pone.0159966.g003

$0.37, \mathrm{P}$ value $=0.46, \mathrm{P}$ for heterogeneity $=0.09, \mathrm{I}^{2}=58 \%$; depression: $\mathrm{SMD}=-0.15,95 \% \mathrm{CI}$ : -0.51 to 0.22 , $\mathrm{P}$ value $=0.28, \mathrm{P}$ for heterogeneity $\left.=0.57, \mathrm{I}^{2}=0 \%\right)($ Fig 6$)$.

We next performed subgroup analyses of adults and children. Among the adults, there were non-significant differences in cardiorespiratory fitness, muscle strength, and fatigue between the exercise and control groups. Among the children, there were non-significant differences in cardiorespiratory fitness and fatigue but a significant difference in muscle strength between the two groups $(\mathrm{SMD}=0.98,95 \%$ CI: 0.40 to 1.55 , $\mathrm{P}$ value $=0.0009$, $\mathrm{P}$ for heterogeneity $=0.89$, $\mathrm{I}^{2}=0 \%$ ) (Fig 7). In addition, subgroup analyses based on induction therapy and post-remission therapy were performed. For induction therapy, there was a non-significant difference in fatigue but significant differences in cardiorespiratory fitness and muscle strength between the exercise and control groups (cardiorespiratory fitness: $\mathrm{SMD}=0.78,95 \% \mathrm{CI}$ : 0.32 to $1.24, \mathrm{P}$ value $=0.0009, \mathrm{P}$ for heterogeneity $=0.63, \mathrm{I}^{2}=0 \%$; muscle strength: $\mathrm{SMD}=0.63,95 \%$ CI: 0.14 


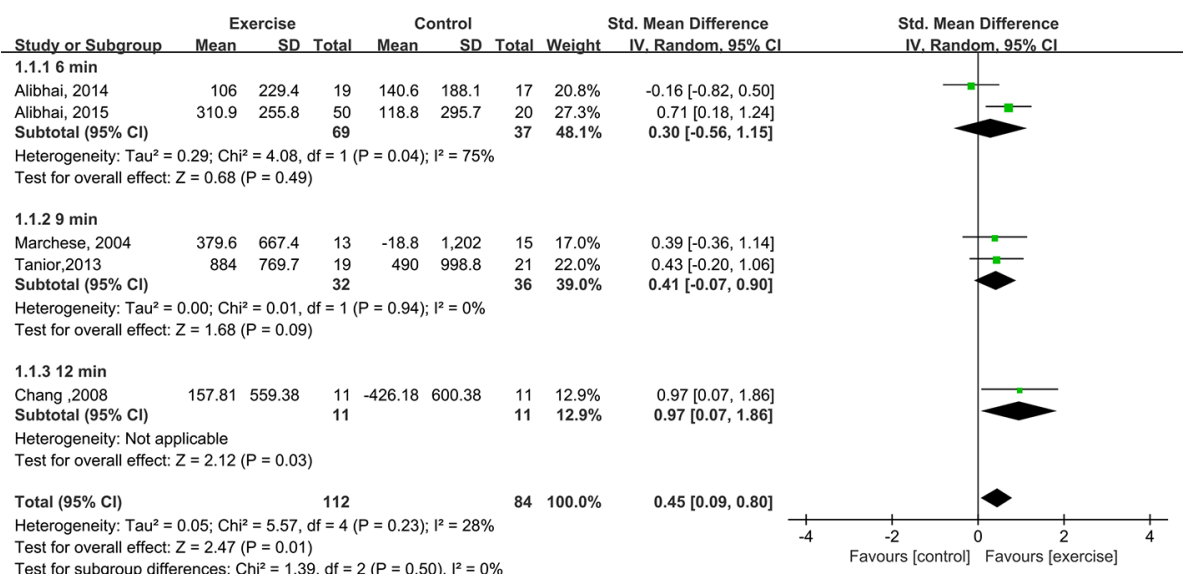

Fig 4. Forest plot comparing the changes in cardiorespiratory fitness between the exercise and control groups.

doi:10.1371/journal.pone.0159966.g004

to 1.12 , P value $=0.01$, heterogeneity: not applicable). For post-remission therapy, there were non-significant differences in cardiorespiratory fitness, muscle strength, and fatigue between the exercise and control groups. Thus, significant differences were found in muscle strength among children with AL undergoing induction therapy and in cardiorespiratory fitness among AL patients undergoing induction therapy regardless of age.

Other outcomes. Functional mobility was measured using the "timed up and down stairs test" and the "timed up and go test" [21,27]. The aggregated results of the "timed up and down stairs test" showed a significant difference between the exercise and control groups, with an MD of -1.52 (95\% CI: -2.38 to -0.65 , $\mathrm{P}$ value $=0.0006$, $\mathrm{P}$ for heterogeneity $=0.20, \mathrm{I}^{2}=39 \%$ ). The results of the "timed up and go test" also revealed a significant difference between the exercise and control groups (SMD $=-1.15,95 \% \mathrm{CI}:-1.83$ to 0.48 , P value $=0.0008)$. Alibhai et al. $[19,20]$ assessed overall health-related QOL using the European Organization for Research and Treatment of Cancer (EORTC) questionnaire for cancer patients (the EORTC-QLQC30), and

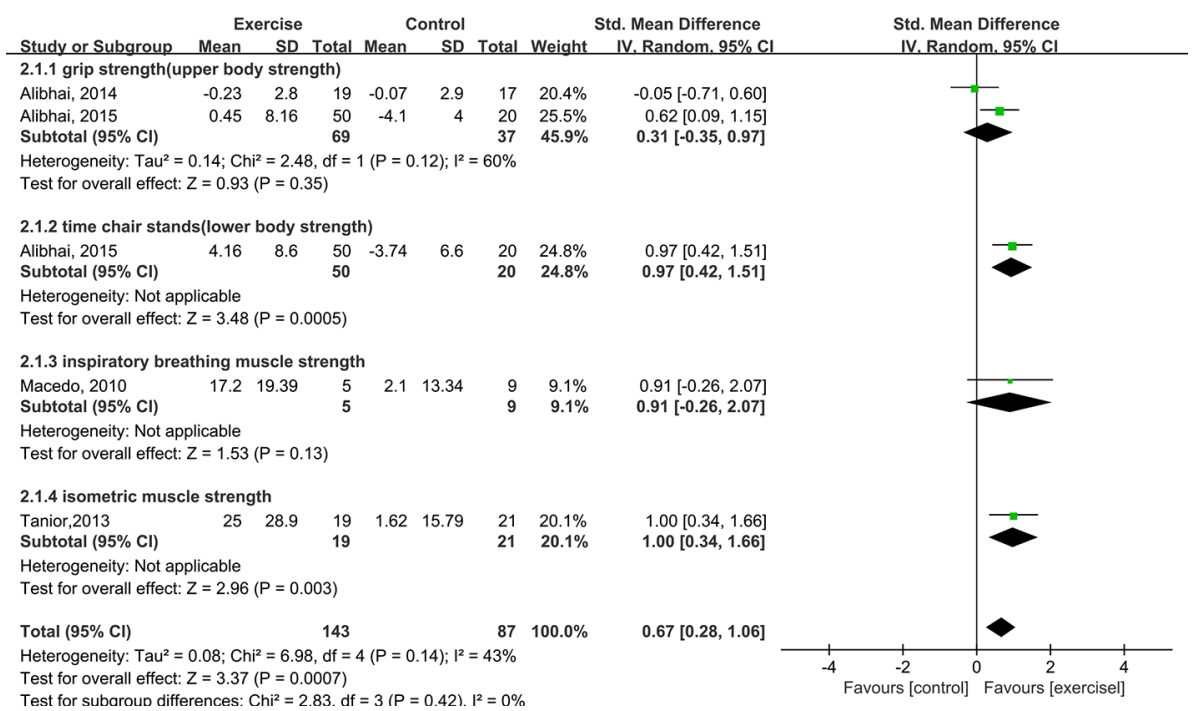

Fig 5. Forest plot comparing the changes in muscle strength between the exercise and control groups.

doi:10.1371/journal.pone.0159966.g005 


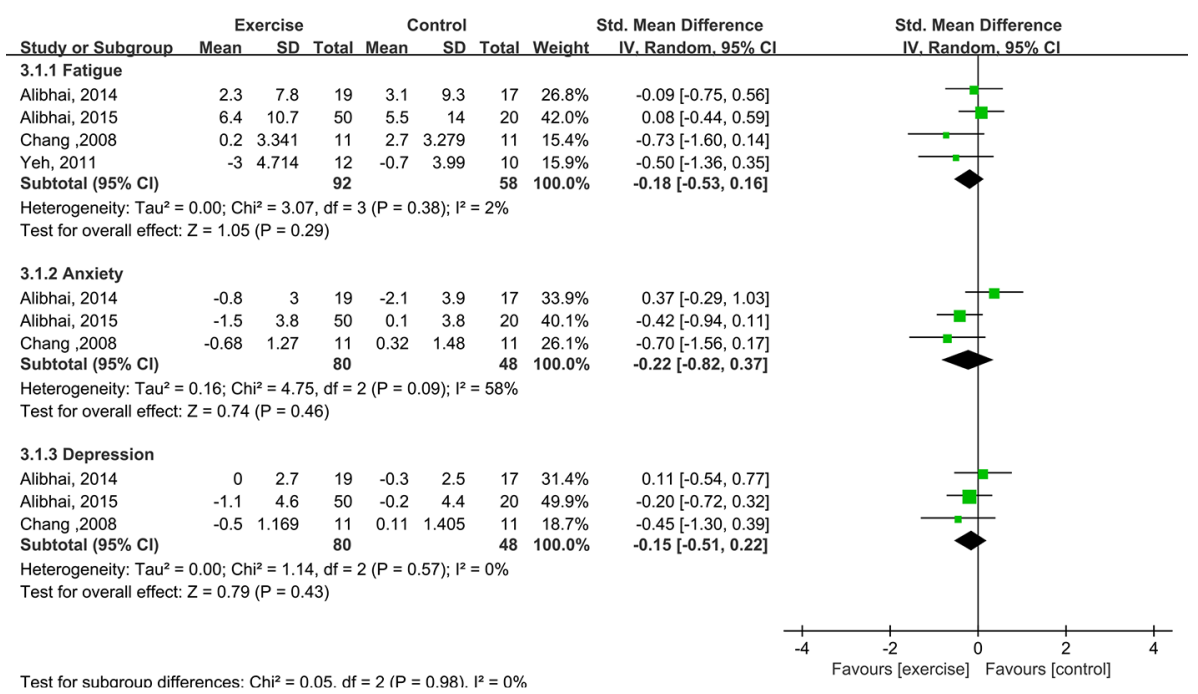

Fig 6. Forest plot comparing the changes in fatigue, anxiety and depression between exercise and control groups.

doi:10.1371/journal.pone.0159966.g006

Marchese et al. [27] assessed QOL using the general Pediatric Quality of Life Inventory ${ }^{\mathrm{TM}}$ Measurement Model (PedQL) 3.0 and the cancer PedQL. However, because the outcome definitions of these instruments differed, we did not pool these data. The aggregated results of Alibhai et al. $[19,20]$ showed a non-significant difference in overall health-related QOL between the exercise and control groups (SMD $=-7.61,95 \% \mathrm{CI}:-16.34$ to $1.11, \mathrm{P}$ value $=0.09$, $\mathrm{P}$ for heterogeneity $=0.31, \mathrm{I}^{2}=3 \%$ ). The cancer and general PedQL scores reported by Marchese et al. [27] also indicated a non-significant difference between the groups. Marchese et al. [27] and Tanir and Kuguoglu [21] measured blood physiological parameters, including the hemoglobin level and hematocrit. They reported a non-significant difference in the SMD in the hemoglobin level between the exercise and control groups (SMD $=-0.29,95 \% \mathrm{CI}:-0.19$ to 0.77 , $\mathrm{P}$ value $=0.23$, $\mathrm{P}$ for heterogeneity $=0.80, \mathrm{I}^{2}=0 \%$ ). In addition, differences in BMI, which was assessed to evaluate body composition, between the two groups were studied in two trials $[24,25]$. The pooled results of these studies showed a non-significant difference in BMI between the exercise and control groups ( $\mathrm{SMD}=0.60,95 \% \mathrm{CI}$ : -0.25 to 1.44 , $\mathrm{P}$ value $=0.17$, $\mathrm{P}$ for heterogeneity $\left.=0.16, \mathrm{I}^{2}=51 \%\right)$.

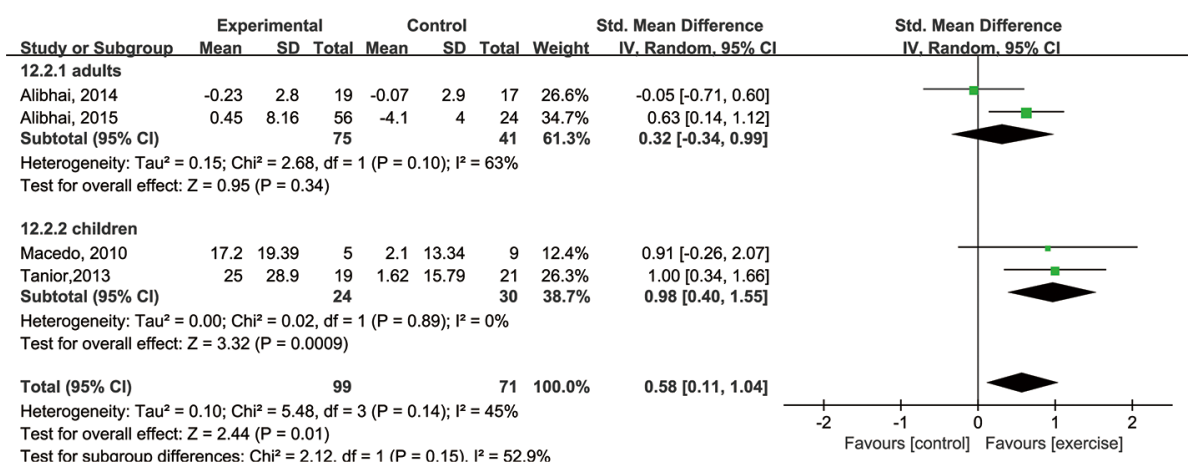

Fig 7. Forest plot of subgroup analyses comparing changes in muscle strength among adults and children.

doi:10.1371/journal.pone.0159966.g007 


\section{Discussion}

This meta-analysis provides a comprehensive summary of the current evidence supporting the efficacy of exercise in AL patients. The results suggest that exercise significantly improves cardiorespiratory fitness and muscle strength. Functional mobility, which was measured using the "timed up and down stairs test" and the "timed up and go test", also showed significant improvement in the exercise group compared to the control group. However, no improvements in fatigue, anxiety, depression, QOL, the hemoglobin level, hematocrit or BMI were observed in the exercise group compared to the control group.

Theoretical evidence of the effects of exercise has emerged. Winningham's Psychobiological-Entropy Model, a theoretical framework for cancer-related fatigue, proposes that a balance between rest and exercise is vitally important for reducing fatigue but that an imbalance could lead to functional deterioration [29]. In addition, the National Comprehensive Cancer Network has stated that exercise reduces cancer-related fatigue by improving the functional capacity, thereby reducing effort and perceived fatigue [30]. Dimeo [31] has also noted that the prevailing recommendations that patients rest and avoid intensive exercise are likely to be counterproductive and lead to muscle wasting, reduced cardiorespiratory fitness and increased fatigue. Several biological mechanisms of the effects of exercise on patients with solid tumors have been reported. Mock [32] has suggested that endorphins, a class of depressants, are secreted via pituitary gland stimulation resulting from exercise, thereby improving the responsiveness of the central nervous system and the body's tolerance to strong stimulation. Simultaneously, the nervous system produced microstimulation during exercise, which relieves muscle tension, anxiety and depression. In addition, exercise enhance metabolism to promote the clearance of metabolic waste and accumulated adrenaline. Exercise also promotes blood circulation, resulting in improved organ function. Thus, exercise has beneficial effects on patients' cardiorespiratory fitness, muscle strength, fatigue, and negative moods.

This is the first meta-analysis to summarize the efficacy of exercise for individuals with AL based on document retrieval. Our results are similar to those of the most recent SR [16]. In detail, that review included five studies demonstrating that exercise interventions appear to be safe and feasible for individuals with AML, although the evidence was insufficient to support the benefits of exercise for chronic myelocytic leukemia patients. However, the conclusions of that SR were limited because of variation in study types, small sample sizes and lack of metaanalysis. Only two studies in that SR were included in our meta-analysis. Other reviews have focused on hematologic malignancies. Bergenthal et al. [3] conducted a Cochrane review evaluating the safety, feasibility and efficacy of aerobic exercise for adults with hematologic malignancies. Liu et al. [14] reviewed ten studies (three RCTs and seven non-RCTs) that examined the effects of exercise on children and adults suffering from hematologic cancers. Further, Wolin et al. [4] investigated the effects of exercise on adult and pediatric survivors of hematologic cancer (including five RCTs and eight non-RCTs). Only 3 RCTs [24,26,27] mentioned in these reviews were included in our meta-analysis. In contrast to the previous reviews, the present meta-analysis showed that exercise significantly improved cardiorespiratory fitness and muscle strength, and these findings are consistent with those of Bergenthal et al. [3]. In this meta-analysis, all studies that assessed fatigue, anxiety and depression showed positive trends of improvements in fatigue and mood due to exercise. However, the aggregated results did not demonstrate a significant difference in fatigue or anxiety between the exercise and control groups, whereas Bergenthal's study did show significant differences in these parameters.

In addition, subgroup analyses according to age and treatment stage were conducted to determine the optimal exercise parameters for individuals with AL. These analyses revealed that exercise significantly improved muscle strength in children with AL undergoing induction 
therapy and cardiorespiratory fitness in AL patients of any age undergoing induction therapy. Additional evidence is needed to validate and expand upon these findings.

To determine the most effective type, intensity and duration of exercise, further trials with more participants and rigorous study designs are needed. The comparability of the study data could also be enhanced by standardizing the measurement instruments. We found that most studies lacked objective outcomes, such as BMI, the hemoglobin level, and peak $\mathrm{VO}_{2}$, as well as safety and feasibility assessments, which would have resulted in more reliable and convincing evidence for clinical applications. In the future, interdisciplinary teams, including basic laboratory scientists, rehabilitation specialists and physical therapists, are expected to participate in further studies.

\section{Limitations}

This meta-analysis has some limitations. One major limitation is the small number of included studies, which restricted our ability to summarize the exercise data for individuals with AL with different characteristics and to examine the efficacy of exercise by conducting subgroup analyses according to the frequency, intensity, time, and type of exercise. For the same reason, a funnel plot could not be generated to evaluate publication bias. Moreover, as an intervention, exercise is typically evaluated by researchers themselves by performing trials because there is no manufacturer or company that can probe for missing data. These factors and missing data may have impacted our results and led to bias.

\section{Conclusion}

In summary, the positive findings of this meta-analysis suggest that exercise has beneficial effects on cardiorespiratory fitness, muscle strength and functional ability. No significant exercise-induced improvements in fatigue, anxiety, depression or QOL were found. AL patients need to exercise in order to counteract side effects (fatigue and myasthenia) with caution due to risk of bleeding. Considering this dilemma, it is necessary to conduct further research on this population. We hope to attract more researchers to this topic so that additional evidence of the efficacy of exercise for AL patients can be obtained. To date, due to the shortage of relevant studies, there is insufficient evidence available to assess the safety, feasibility and efficacy of exercise programs for AL patients. Therefore, high-quality RCTs examining exercise interventions are imperative for this population.

\section{Author Contributions}

Conceived and designed the experiments: YZ ZG. Performed the experiments: YZ JZ ZG XY. Analyzed the data: YZ JZ. Contributed reagents/materials/analysis tools: YZ JZ. Wrote the paper: YZZG.

\section{References}

1. Pate RR, Pratt M, Blair SN, Haskell WL, Macera CA, Bouchard C, et al. Physical activity and public health. A recommendation from the Centers for Disease Control and Prevention and the American College of Sports Medicine. JAMA. 1995; 273: 402-407. PMID: 7823386

2. Danaher EH, Ferrans C, Verlen E, Ravandi F, van Besien K, Gelms J, et al. Fatigue and physical activity in patients undergoing hematopoietic stem cell transplant. Oncol Nurs Forum. 2006; 33: 614-624. doi: 10.1188/06.ONF.614-624 PMID: 16676017

3. Bergenthal N, Will A, Streckmann F, Wolkewitz K-D, Monsef I, Engert A, et al. Aerobic physical exercise for adult patients with haematological malignancies. Cochrane Database Syst Rev. 2014. doi: 10.1002/ 14651858.CD009075.pub2 
4. Wolin $\mathrm{KY}$, Ruiz JR, Tuchman $\mathrm{H}$, Lucia A. Exercise in adult and pediatric hematological cancer survivors: an intervention review. Leukemia. 2010; 24: 1113-1120. doi: 10.1038/leu.2010.54 PMID: 20410923

5. San Juan AF, Chamorro-Vina C, Moral S, Fernandez del Valle M, Madero L, Ramirez M, et al. Benefits of intrahospital exercise training after pediatric bone marrow transplantation. Int J Sports Med. 2008; 29: 439-446. doi: 10.1055/s-2007-965571 PMID: 17960520

6. De Souza AM, Potts JE, Potts MT, De Souza ES, Rowland TW, Pritchard SL, et al. A stress echocardiography study of cardiac function during progressive exercise in pediatric oncology patients treated with anthracyclines. Pediatr Blood Cancer. 2007; 49: 56-64. doi: 10.1002/pbc.21122 PMID: 17226848

7. Krull KR, Annett RD, Pan Z, Ness KK, Nathan PC, Srivastava DK, et al. Neurocognitive functioning and health-related behaviours in adult survivors of childhood cancer: a report from the Childhood Cancer Survivor Study. Eur J Cancer. 2011; 47: 1380-1388. doi: 10.1016/j.ejca.2011.03.001 PMID: 21458986

8. Aznar S, Webster AL, San Juan AF, Chamorro-Vina C, Mate-Munoz JL, Moral S, et al. Physical activity during treatment in children with leukemia: a pilot study. Appl Physiol Nutr Metab. 2006; 31: 407-413. doi: 10.1139/h06-014 PMID: 16900230

9. Schule K. [The rank value of sports and movement therapy in patients with breast or pelvic cancer]. Rehabilitation (Stuttg). 1983; 22: 36-39.

10. Courneya KS, McKenzie DC, Mackey JR, Gelmon K, Friedenreich CM, Yasui Y, et al. Effects of exercise dose and type during breast cancer chemotherapy: multicenter randomized trial. J Natl Cancer Inst. 2013; 105: 1821-1832. doi: 10.1093/jnci/djt297 PMID: 24151326

11. Doyle C, Kushi LH, Byers T, Courneya KS, Demark-Wahnefried W, Grant B, et al. Nutrition and physical activity during and after cancer treatment: an American Cancer Society guide for informed choices. CA Cancer J Clin. 2006; 56: 323-353. PMID: 17135691

12. Dieli-Conwright CM, Mortimer JE, Schroeder ET, Courneya K, Demark-Wahnefried W, Buchanan TA, et al. Randomized controlled trial to evaluate the effects of combined progressive exercise on metabolic syndrome in breast cancer survivors: rationale, design, and methods. BMC Cancer. 2014; 14: 238. doi: 10.1186/1471-2407-14-238 PMID: 24708832

13. McCullough DJ, Stabley JN, Siemann DW, Behnke BJ. Modulation of blood flow, hypoxia, and vascular function in orthotopic prostate tumors during exercise. J Natl Cancer Inst. 2014; 106: dju036. doi: 10. 1093/jnci/dju036 PMID: 24627275

14. Liu RDKS, Chinapaw MJM, Huijgens PC, van Mechelen W. Physical exercise interventions in haematological cancer patients, feasible to conduct but effectiveness to be established: a systematic literature review. Cancer Treat Rev. 2009; 35: 185-192. doi: 10.1016/j.ctrv.2008.09.008 PMID: 19004560

15. Jochem C, Leitzmann MF, Keimling M, Schmid D, Behrens G. Physical activity in relation to risk of hematologic cancers: a systematic review and meta-analysis. Cancer Epidemiol Biomarkers Prev. 2014; 23: 833-846. doi: 10.1158/1055-9965.epi-13-0699 PMID: 24633143

16. Smith-Turchyn J, Richardson J. A systematic review on the use of exercise interventions for individuals with myeloid leukemia. Support Care Cancer. 2015; 23: 2435-2446. doi: 10.1007/s00520-015-2752-3 PMID: 25947256

17. Siegel RL, Miller KD, Jemal A. Cancer statistics, 2016. CA Cancer J Clin. 2016; 66: 7-30. doi: 10.3322/ caac.21332 PMID: 26742998

18. Sallan SE. Myths and lessons from the adult/pediatric interface in acute lymphoblastic leukemia. Hematology Am Soc Hematol Educ Program. 2006: 128-132. doi: 10.1182/asheducation-2006.1.128 PMID: 17124051

19. Alibhai SM, Durbano S, Breunis H, Brandwein JM, Timilshina N, Tomlinson GA, et al. A phase II exercise randomized controlled trial for patients with acute myeloid leukemia undergoing induction chemotherapy. Leuk Res. 2015. doi: 10.1016/j.leukres.2015.08.012

20. Alibhai SMH, O'Neill S, Fisher-Schlombs K, Breunis H, Timilshina N, Brandwein JM, et al. A pilot phase II RCT of a home-based exercise intervention for survivors of AML. Support Care Cancer. 2014; 22 : 881-889. doi: 10.1007/s00520-013-2044-8 PMID: 24240647

21. Tanir MK, Kuguoglu S. Impact of exercise on lower activity levels in children with acute lymphoblastic leukemia: a randomized controlled trial from Turkey. Rehabil Nurs. 2013; 38: 48-59. doi: 10.1002/rnj.58 PMID: 23365005

22. Higgins JP, Altman DG, Gotzsche PC, Juni P, Moher D, Oxman AD, et al. The Cochrane Collaboration's tool for assessing risk of bias in randomised trials. BMJ. 2011; 343: d5928. doi: 10.1136/bmj. d5928 PMID: 22008217

23. De Macedo TMF, Oliveira KMC, Melo JBDC, De Medeiros MG, De Medeiros FWC, Ferreira GMH, et al. Inspiratory muscle training in patients with acute leukemia: Preliminary results. Revista Paulista de Pediatria. 2010; 28: 352-358. 
24. Hartman A, Winkel MLt, van Beek RD, Keizer-Schrama SMPFdM, Kemper HCG, Hop WCJ, et al. A randomized trial investigating an exercise program to prevent reduction of bone mineral density and impairment of motor performance during treatment for childhood acute lymphoblastic leukemia. Pediatr Blood Cancer. 2009; 53: 64-71. doi: 10.1002/pbc.21942 PMID: 19283791

25. Moyer-Mileur LJ, Ransdell L, Bruggers CS. Fitness of children with standard-risk acute lymphoblastic leukemia during maintenance therapy response to a home-based exercise and nutrition program. $J$ Pediatr Hematol Oncol. 2009; 31: 259-266. doi: 10.1097/MPH.0b013e3181978fd4 PMID: 19346877

26. Chang PH, Lai YH, Shun SC, Lin LY, Chen ML, Yang Y, et al. Effects of a walking intervention on fatigue-related experiences of hospitalized acute myelogenous leukemia patients undergoing chemotherapy: a randomized controlled trial. J Pain Symptom Manage. 2008; 35: 524-534. doi: 10.1016/j. jpainsymman.2007.06.013 PMID: 18280104

27. Marchese VG, Chiarello LA, Lange BJ. Effects of physical therapy intervention for children with acute lymphoblastic leukemia. Pediatr Blood Cancer. 2004; 42: 127-133. doi: 10.1002/pbc.10481 PMID: 14752875

28. Yeh CH, Wai JPM, Lin U-S, Chiang Y-C. A Pilot study to examine the feasibility and effects of a homebased aerobic program on reducing fatigue in children with acute lymphoblastic leukemia. Cancer Nurs. 2011; 34: 3-12. doi: 10.1097/NCC.0b013e3181e4553c PMID: 20706112

29. Winningham $M$. How exercise mitigates fatigue: implications for people receiving cancer therapy. Biotherapy of Cancer. 1992: 16-21.

30. Berger AM, Mooney K, Alvarez-Perez A, Breitbart WS, Carpenter KM, Cella D, et al. Cancer-related fatigue, version 2.2015. J Natl Compr Canc Netw. 2015; 13: 1012-1039. PMID: 26285247

31. Dimeo FC. Effects of exercise on cancer-related fatigue. Cancer. 2001; 92: 1689-1693. PMID: 11598888

32. Mock V. Breast cancer and fatigue: issues for the workplace. AAOHN J. 1998; 46: 425-431; quiz 432423. PMID: 9923203 